\title{
Limit of Spatial Resolution of Refractometer Cavities
}

\author{
William J. Hartman
}

(July 9, 1959)

\begin{abstract}
Filter factors that determine an upper limit for the wave numbers for which refractometer measurements can be used to calculate the spectrum of refractivity are derived in this paper based on the assumption that refractometers measure a weighted average of the refractive index in a volume of air surrounding the center of the refractometer cavity. Two models are used assuming the weighting function has spherical symmetry around the center and one model is used assuming the function has cylindrical symmetry. All models result in a simple mathematical form which should be easy to use in further theoretical developments.
\end{abstract}

\section{Introduction}

The wave number power spectrum of refractivity is determined for large wave numbers $k$ by the correlation of the refractive index variations at points which are separated by small distances [1]. ${ }^{1}$ Thus, refractometer measurements discriminate in two ways against the spectrum for large wave numbers. First, the refractometer does not measure the variation of the refractive index at a point, but rather, a weighted average of the variations in the volume of air in the vicinity of the center of the refractometer cavity, and second, the centers of two cavities may not be placed arbitrarily close because of obvious physical limitations. The first of these effects is evaluated in this paper assuming several different weighting functions and neglecting further practical limitations such as finite response time and noise characteristies of the instrument. The result shows that the measured spectrum is a filter factor times the theoretical spectrum. With appropriate approximations, the effect is also evaluated for the measured frequency spectrum of refractivity.

The exact weighting function is determined by, among other things, the size and shape of the cavity and the size and shape of the openings which allow the passage of air through the cavity. The commonly used microwave refractometer cavity [2] consists of a right circular cylinder with the ends partially opened to permit the flow of air and operated in the $T E_{011}$ mode at about $9 \mathrm{kMc}$. Considering the weighting function for a closed cylindrical cavity operated in the $T E_{011}$ mode, it appears that the weighting function for an open cavity with the same dimensions, and operated in the same mode, decreases approximately as a partial sine wave across a section of the cavity perpendicular to the axis and gaussianly along the axis from the center [3]. For mathematical simplicity, however, the most desirable weighting function is one that has spherical symmetry about the center of the cavity, as will be seen later in the analysis in this paper. (This would be a very good approximation, for example, for a refractometer using a cavity consisting of two concentric spheres, the outer sphere being a grid to allow for the passage of air.) Two of the weighting functions used in this paper have spherical symmetry, and a third function is used which has cylindrical symmetry. The results for the three different forms differ significantly only in the region where data become unreliable because of other limitations.

\section{Theoretical Development}

It may be assumed that the refractometer measures a weighted spatial average of the variation $\Delta n=n-n_{0}$ where $n_{0}$ denotes an average value of the refractive index $n$. Let the

1 Figures in brackets indicate the literature references at the end of this paper. 
weighted average of $\Delta n$ at any time $t$ be given by

$$
\overline{\Delta n}(\vec{R}, t)=\frac{\int_{v} d^{3} r \Delta n \overrightarrow{\left(r^{\prime}, t\right) f(\vec{r})}}{\int_{v} d^{3} r \overrightarrow{f(r)}}=\frac{\int_{v} d^{3} r \Delta n(\vec{R}+\vec{r}, t) \overrightarrow{f(r)}}{\int_{v} d^{3} r f(\vec{r})}
$$

where $v$ is a volume centered at $\vec{R}$, the center of the refractometer cavity, and $\overrightarrow{f(r)}$ is the weighting function for the cavity. Figure 1 shows two spherical cavities and the vectors associated with these.

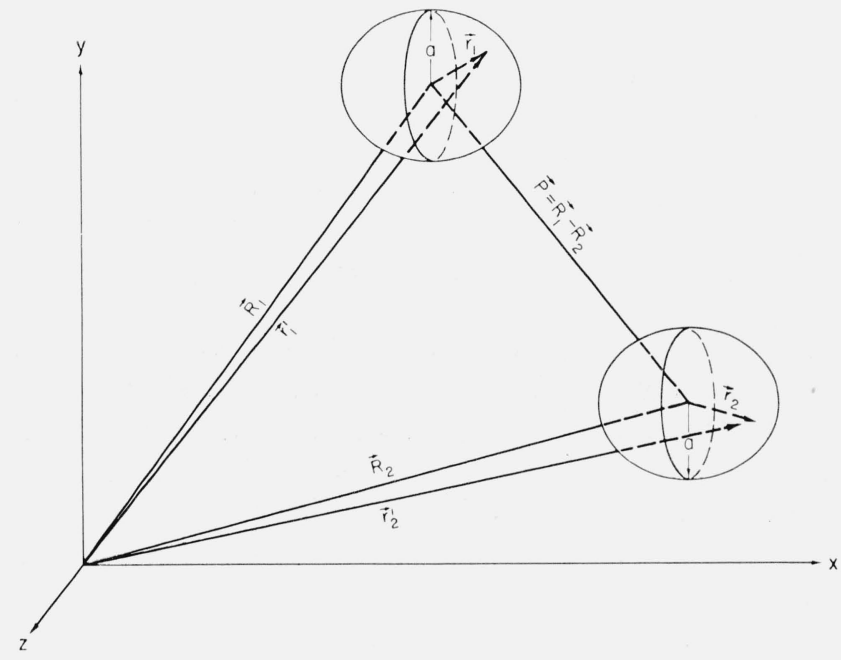

Figure 1. Geometry for two spherical refractometer cavities.

Using two refractometers with centers at $\vec{R}_{1}$ and $\vec{R}_{2}$, it follows that

$$
\left\langle\overline{\Delta n}\left(\vec{R}_{1}, t\right) \overline{\Delta n}\left(\vec{R}_{2}, t\right)\right\rangle=\frac{\int_{v_{1}} d^{3} r_{1} \int_{v_{2}} d^{3} r_{2}\left\langle\Delta n\left(\vec{r}_{1}^{\prime}, t\right) \Delta n\left(\vec{r}_{2}^{\prime}, t\right)>f\left(\overrightarrow{r_{1}}\right) f\left(\vec{r}_{2}\right)\right.}{\int_{v_{1}} d^{3} r_{1} \int_{v_{2}} d^{3} r_{2} f\left(\overrightarrow{r_{1}}\right) f\left(\vec{r}_{2}\right)}
$$

where $\langle>$ denotes a time average or ensemble average.

Following Wheelon $[4]^{2}$, one obtains

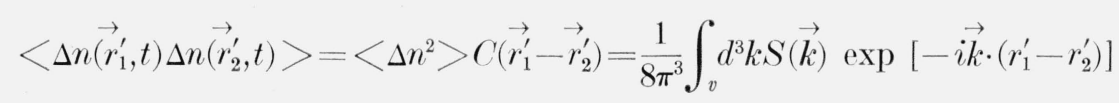

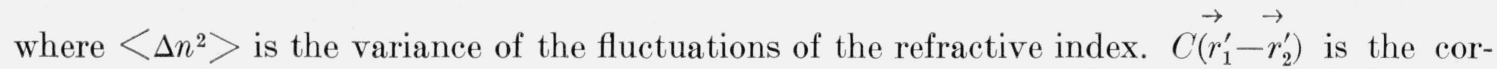
relation of the fluctuations at two points determined by the vectors $\overrightarrow{r_{1}^{\prime}}$ and $\overrightarrow{r_{2}^{\prime}}$, and $\left.S \vec{k}\right)$ is the wave number power spectrum of refractivity.

Similarly, one may define

$$
\left\langle\overline{\Delta n}\left(\vec{R}_{1}, t\right) \overline{\Delta n}\left(\vec{R}_{2}, t\right)>=\left\langle\Delta n^{2}>C_{w}(\vec{P})\right.\right.
$$

where $C_{w}(\vec{P})$ is the correlation function between the centers of the cavities of two refractometers, and the vector $\vec{P}$ is the vector between the two centers, $\vec{P}=\vec{R}_{1}-\vec{R}_{2}$.

${ }^{2}$ The magnitude of the $S(k)$ defined by Wheelon is about one-fourth as large as the one used here since he used $\Delta \epsilon \cong 2 n \Delta n$ instead of $\Delta n$. 
Combining (2), (3), and (4) one finds that

$$
\begin{aligned}
& \left\langle\overline{\Delta n}\left(\vec{R}_{1}, t\right) \overline{\Delta n}\left(\vec{R}_{2}, t\right)\right\rangle=\left\langle\Delta n^{2}>C_{w}(\vec{P})=\frac{\int_{v_{1}} d^{3} r_{1} \int_{v_{2}} d^{3} r_{2}<\Delta n^{2}>C\left(\overrightarrow{r_{1}^{\prime}-}-\vec{r}_{2}^{\prime}\right) f\left(\overrightarrow{r_{1}}\right) f\left(\overrightarrow{r_{2}}\right)}{\int_{v_{1}} d^{3} r_{1} \int_{v_{2}} d^{3} r_{2} f\left(\overrightarrow{r_{1}}\right) f\left(\overrightarrow{r_{2}}\right)}\right. \\
& =\frac{\int_{v_{1}} d^{3} r_{1} \int_{v_{2}} d^{3} r_{2} f\left(\overrightarrow{r_{1}}\right) f\left(\overrightarrow{r_{2}}\right)\left[\frac { 1 } { 8 \pi ^ { 3 } } \int _ { v } d ^ { 3 } k \operatorname { e x p } \left[-\overrightarrow{i k} \cdot\left(\overrightarrow{\left.\left.\left.r_{1}{ }^{\prime}-\vec{r}_{2}{ }^{\prime}\right)\right] S(\vec{k})\right]}\right.\right.\right.}{\int_{v_{1}} d^{3} r_{1} \int_{v_{2}} d^{3} r_{2} f\left(\overrightarrow{r_{1}}\right) f\left(\overrightarrow{r_{2}}\right)} \\
& =\frac{1}{8 \pi^{3}} \int_{v} d^{3} k \exp [-\overrightarrow{i k} \cdot \vec{P}] S(\vec{k})\left\{\frac{\int_{v_{1}} d^{3} r_{1} \int_{v_{2}} d^{3} r_{2} f\left(\vec{r}_{1}\right) f\left(\vec{r}_{2}\right) \exp \left[-i \vec{k} \cdot\left(\overrightarrow{r_{1}}-\vec{r}_{2}\right)\right]}{\int_{v_{1}} d^{3} r_{1} \int_{v_{2}} d^{3} r_{2} f\left(\vec{r}_{1}\right) f\left(\vec{r}_{2}\right)}\right\} .
\end{aligned}
$$

The factor in the braces in (5) is called the filter factor $E$ and this determines the upper limit of $k$ for which the refractometer may be used to measure $S \overrightarrow{(k)}$. It should be noted that in the above formulation, the exact form of the structure of the atmospheric turbulence, whether isotropic or anisotropic, affects the weighting function but will have no other effect on $E$. In practice, $P$ must be greater than the size of the cavity, but for theoretical purposes, one may assume that $P$ can take on any value greater than or equal to zero. Then, taking the inverse Fourier transform of (5), one finds

$$
\operatorname{ES}(\vec{k})=\int_{v} d^{3} P \exp [\overrightarrow{i k} \cdot \vec{P}]<\Delta n^{2}>C_{w}(\vec{P})
$$

which is the quantity obtained from measurements.

In the following we will evaluate $E$ assuming the following forms for $\mathrm{f}(\overrightarrow{\mathrm{r}})$ :

and

$$
\begin{aligned}
& 1^{\circ} \overrightarrow{f(r)}=\left\{\begin{array}{l}
1 \text { for }|\vec{r}| \leq a, \\
0 \text { for }|\vec{r}|>a
\end{array}\right. \\
& 2^{\circ} \overrightarrow{f(r)}=\exp \left\{-r^{2} / a^{2}\right\},
\end{aligned}
$$

$$
3^{\circ} \overrightarrow{f(r)}= \begin{cases}\exp \left\{-r_{x}^{2} / b^{2}\right\} & \text { for } \sqrt{r_{y}^{2}+r_{z}^{2}} \leq a \\ 0 & \text { for } \sqrt{r_{y}^{2}+r_{z}^{2}}>a\end{cases}
$$

where $r_{x}, r_{y}$, and $r_{z}$ are the components of $\vec{r}$ in the $x, y$, and $z$ directions respectively. The first form, $1^{\circ}$, gives an upper bound for any closed spherical refractometer cavity of radius $a$, and the second form, $2^{\circ}$, gives an upper bound for such a cavity operated in the $T E_{011}$ mode. In each of the three forms it is possible (but not necessary) to identify the parameter $a$ (or $b$ ) with the dimension of the cavity.

For the first case, $1^{\circ}$, if one has two refractometers which measure a small spherical volume of air of radius $a$ and $b$, respectively

$$
\begin{aligned}
E=\left\{\frac{1}{\left(\frac{4}{3} \pi a^{3}\right)} \int_{0}^{\pi} \sin \lambda_{1} d \lambda_{1} \int_{0}^{2 \pi} d \theta_{1} \int_{0}^{a} r_{1}^{2} d r_{1} \exp \left[-i k r_{1} \cos \lambda_{1}\right]\right\} \\
\\
\qquad\left\{\frac{1}{\left(\frac{4}{3} \pi b^{3}\right)} \int_{0}^{\pi} \sin \lambda_{2} d \lambda_{2} \int_{0}^{2 \pi} d \theta_{2} \int_{0}^{b} r_{2}^{2} d r_{2} \exp \left[i k r_{2} \cos \lambda_{2}\right]\right\} .
\end{aligned}
$$


The quantities in the braces in (7) are the same, except that the parameter $(a)$ is changed and one is the complex conjugate of the other. Thus we may write

$$
E=g(k, a) g^{*}(k, b)
$$

where $g^{*}(k, b)$ is the complex conjugate of $g(k, b)$. One then obtains by integrating

$$
g(k, a)=\frac{3}{(k a)^{3}}[\sin k a-k a \cos k a]
$$

and thus,

$$
E=\frac{9}{(k a)^{3}(k \bar{b})^{3}}[\sin k a-k a \cos k a][\sin k b-k b \cos k b] .
$$

For the second case, $2^{\circ}$, one finds that

$$
\begin{aligned}
E=\left\{\frac{1}{a^{3} \pi^{3 / 2}} \int_{0}^{\pi} \sin \lambda_{1} d \lambda_{1}\right. & \left.\int_{0}^{2 \pi} d \theta_{1} \int_{0}^{\infty} r_{1}^{2} d r_{1} \exp \left[-i k r_{1} \cos \lambda_{1}\right] \exp \left\{-r_{1}^{2} / a^{2}\right\}\right\} \\
& \times\left\{\frac{1}{b^{3} \pi^{3 / 2}} \int_{0}^{\pi} \sin \lambda_{2} d \lambda_{2} \int_{0}^{2 \pi} d \theta_{2} \int_{0}^{\infty} r_{2}^{2} d r_{2} \exp \left[i k r_{2} \cos \lambda_{2}\right] \exp \left\{-r_{2}^{2} / b^{2}\right\}\right\} .
\end{aligned}
$$

Again, one may set the two factors of the right hand side of $(11)$ equal respectively to $g(k, a)$ and $g^{*}(k, b)$. After performing the $\lambda$ and $\theta$ integrations, one obtains

$$
g(k, a)=\frac{4}{a^{3} \sqrt{\pi}} \int_{0}^{\infty} \frac{\sin k r}{k r} r^{2} \exp \left\{-r^{2} / a^{2}\right\} d r
$$

which yields, using relation No. 19, page 73, of reference[5],

$$
g(k, a)=\frac{4}{a^{3} \sqrt{\pi}} \frac{a^{3} \sqrt{\pi}}{4} \frac{k}{k} \exp \left\{-\frac{a^{2} k^{2}}{4}\right\}=\exp \left\{-\frac{a^{2} k^{2}}{4}\right\} .
$$

Thus

$$
E=\exp \left\{-k^{2}\left(a^{2}+b^{2}\right) / 4\right\} .
$$

Figure 2 shows the filter factors for the sharp spherical cutoff $E_{s}(k a)$ and for the gaussian cutoff $E_{g}(k a)$ versus $k a$, assuming that the refractometers used to determine $E_{s}$ (or $E_{g}$ ) are the same.

For the case $3^{\circ}, E$ is given by

$$
E=g(a, b) g^{*}(c, d)
$$

where

$$
g(a, b)=\frac{\int_{-\infty}^{\infty} d r_{x} \int_{-a}^{a} d r_{y} \int_{-L}^{L} d r_{z} e^{-\overrightarrow{i k \cdot r}} \exp \left\{-r_{x}^{2} / b^{2}\right\}}{\int_{-\infty}^{\infty} d r_{x} \int_{-a}^{a} d r_{y} \int_{-L}^{L} d r_{z} \exp \left\{-r_{x}^{2} / b^{2}\right\}}
$$

with $L=\sqrt{a^{2}-r_{y}{ }^{2}}$ and $g^{*}(c, d)$ is the complex conjugate of $g(c, d)$. After integrating the denominator of (15) one obtains

$$
g(a, b)=\frac{1}{\pi^{\frac{3}{2}} a^{2} b} \int_{-\infty}^{\infty} d r_{x} \int_{-a}^{a} d r_{y} \int_{-L}^{L} d r_{z} \exp \left[-i\left(r_{x} k_{x}+r_{y} k_{y}+r_{z} k_{z}\right)\right] \exp \left\{-r_{x}^{2} / b^{2}\right\} .
$$




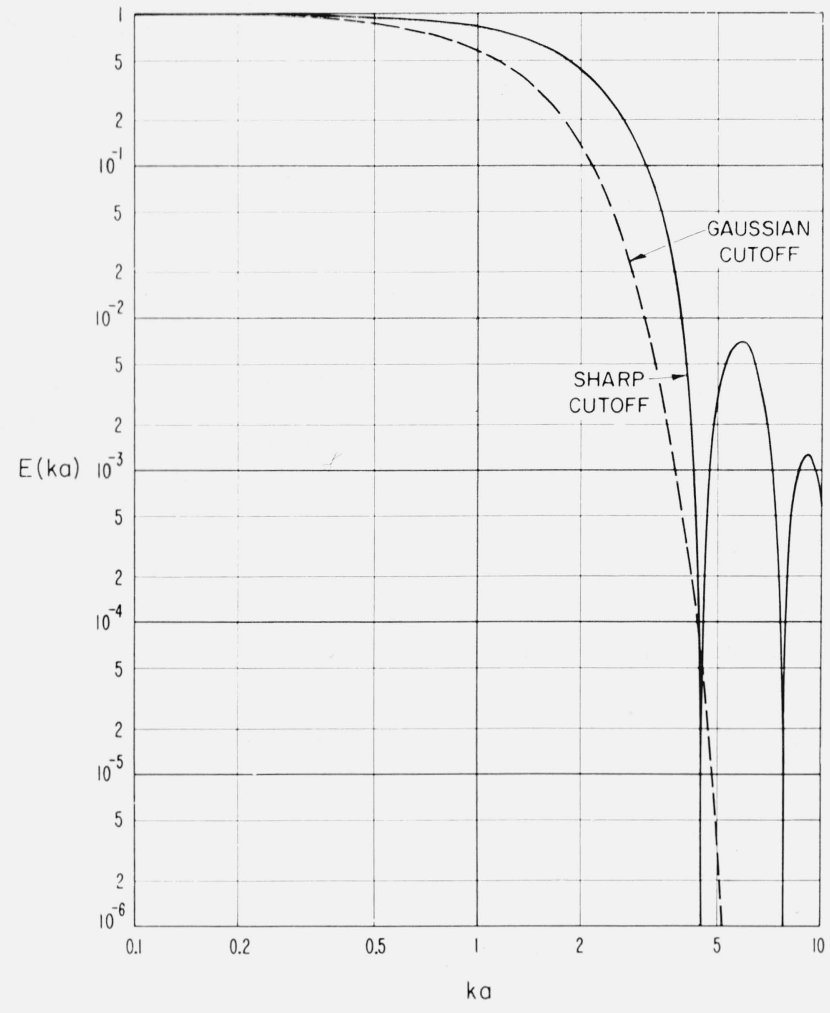

Figure 2. Filter factors for gaussian cutoff and sharp cutoff.

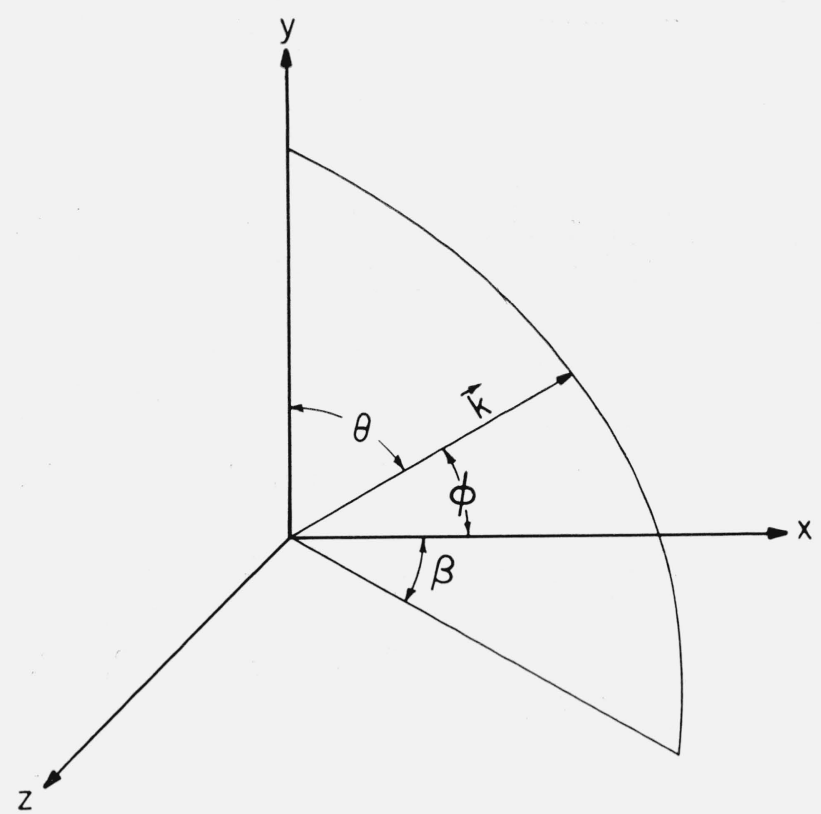

FIGURE 3. Geometry for locating the propagation vector $\vec{k}$ in relation to a cylindrical cavity with its axis parallel to the $x$-axis.

The $r_{x}$ integration may be done using relation No. 23, page 121 in reference [5] and one obtains

$$
g(a, b)=\frac{\exp \left\{-k_{x}^{2} b^{2} / 4\right\}}{\pi a^{2}} \int_{-a}^{a} d r_{y} \int_{-L}^{L} d r_{z} \exp \left\{-i\left(r_{y} k_{y}+r_{z} k_{z}\right)\right\}
$$

Changing to polar coordinates, (17) becomes

$$
g(a, b)=\frac{\exp \left\{-k_{x}^{2} b^{2} / 4\right\}}{\pi a^{2}} \int_{0}^{a} y d y \int_{0}^{2 \pi} d \theta \exp \left[-i y \sqrt{k_{y}{ }^{2}+k_{z}}{ }^{2} \sin \theta\right],
$$

which may be integrated to yield

$$
g(a, b)=\frac{2}{a \sqrt{k_{y}^{2}+k_{z}^{2}}} J_{1}\left(a \sqrt{k_{y}^{2}+k_{z}^{2}}\right) \exp \left[\frac{-k_{x}^{2} b^{2}}{4}\right]
$$

where $J_{1}(Z)$ is the Bessel function of order 1. Referring to figure 3, one finds that

$$
\sqrt{k_{y}{ }^{2}+k_{z}{ }^{2}}=k \sqrt{1-\sin ^{2} \theta \cos ^{2} \beta}
$$

and

$$
k_{x}^{2}=k^{2} \sin ^{2} \theta \cos ^{2} \beta
$$

where $\theta$ is the angle which $\vec{k}$ makes with the vertical, and $\beta$ is the angle in the $X Z$ plane with the $x$-axis assuming that the cavities are both alined along the $x$-axis. Combining (14), (19), 
(20a), and (20b) it follows that

$$
\begin{aligned}
& E_{c}=\frac{4}{a c k^{2}\left(1-\sin ^{2} \theta \cos ^{2} \beta\right)} J_{1}\left(a k \sqrt{1-\sin ^{2} \theta \cos ^{2} \beta}\right) J_{1}\left(c k \sqrt{1-\sin ^{2} \theta \cos ^{2} \beta}\right) \\
& \times \exp \left\{-\frac{\left(\sin ^{2} \theta \cos ^{2} \beta\right) k^{2}\left(b^{2}+d^{2}\right)}{4}\right\} .
\end{aligned}
$$

$E_{c}$ is plotted versus $k a$ in figure 4 , assuming two identical cavities, and also that $a$ equals $b$ in (21a), for the values $\beta$ equal zero and several values of $\theta$.

One may also write

$$
\sqrt{1-\sin ^{2} \theta \cos ^{2} \beta}=\cos \phi
$$

where $\phi$ is the angle $\vec{k}$ forms with the $x$-axis. With this notation, (21a) becomes

$$
E_{c}=\frac{4}{a c k^{2} \cos ^{2} \phi} J_{1}(a k \cos \phi) J_{1}(c k \cos \phi) \exp \left\{-\frac{k^{2}\left(b^{2}+d^{2}\right) \sin ^{2} \phi}{4}\right\}
$$

In figure 4 , the curves for $\theta$ equal to $0^{\circ}, 45^{\circ}$, and $90^{\circ}$ correspond to $\phi$ equal to $90^{\circ}, 45^{\circ}$ and $0^{\circ}$, respectively.

If one assumes isotropic turbulence and then carries out the angular integrations in (5) using the filter factor (21a), a new filter factor is obtained which, as in the two previous forms, is dependent only on the magnitude but not on the direction of $\vec{k}$.

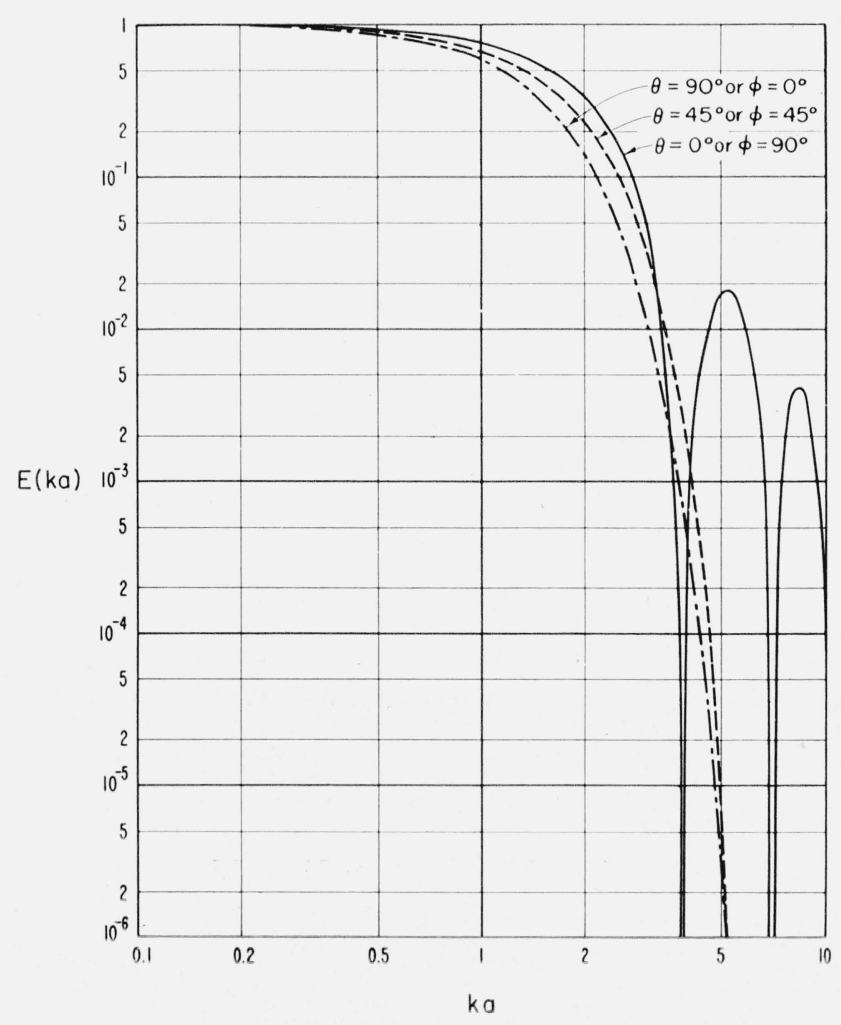

FIGURE 4. Filter factor for a cylindrical refractometer cavity with its axis in the horizontal direction.

$\theta$ is the angle a vector $\vec{k}$, in the $x y$ plane makes with the vertical axis, and the angle $\phi$ is the angle a vector $\vec{k}$ makes with the axis of the cylinder. 
It may be noted here that zeros of the functions shown in figure 2 and figure 4 can actually occur, the only requirement being that the weighting function for the refractometer decrease slowly over a small volume surrounding the center of the cavity.

The frequency spectrum of refractivity is given by [6]

$$
W_{n}(\nu)=\int_{0}^{\infty} d \tau \cos \nu \tau<\Delta n\left(\vec{r}_{1}, t\right) \Delta n\left(\vec{r}_{1}, t+\tau\right)>
$$

Many measurements of this type, taken with a stationary refractometer, are available [2] Assuming (somewhat arbitrarily) that the structure of the turbulence is frozen in blobs which are carried past the refractometer by the wind with the constant velocity $\vec{\mu}$, one may write

$$
\left.\left.\left.\left\langle\Delta n \vec{r}_{1}, t\right) \Delta n \vec{r}_{1}, t+\tau\right)\right\rangle=\left\langle\Delta n \overrightarrow{\left.r_{1}, t\right) \Delta n^{\prime}} \vec{r}_{1}+\vec{\mu} \tau, t\right)\right\rangle,
$$

and then, using (1), it follows that

$$
\left\langle\overline{\Delta n}\left(\vec{r}_{1}, t\right) \overline{\Delta n}\left(\vec{r}_{1}, t+\tau\right)\right\rangle=\left\langle\overline{\Delta n}\left(\vec{r}_{1}, t\right) \overline{\Delta n}\left(\vec{r}_{1}+\overrightarrow{\mu \tau}, t\right)\right\rangle
$$

It should be noted that in the above expressions $((23)$ and $(24))$ no allowance is made for the "self motion" [4] of the air, and this may be of considerable importance. Applying (24) and (5) in (22), one obtains

$$
W_{n a}(\nu)=\int_{0}^{\infty} d \tau \cos \nu \tau \frac{1}{8 \pi^{3}} \int_{v} d^{3} k \exp [-\overrightarrow{i k} \cdot \overrightarrow{\mu \tau}] S(\vec{k}) E(k a),
$$

where $W_{n a}(\nu)$ is the spectrum which is actually measured and $\nu=2 \pi f$ where $f$ is a frequency. For the case of isotropic turbulence (25) yields

$$
W_{n a}(\nu)=\frac{1}{4 \pi \mu} \int_{\nu / \mu}^{\infty} d k k S(k) E(k a),
$$

using either $1^{\circ}$ or $2^{\circ}$ for the filter factor.

Substituting $\nu=k \mu$ in (26) and differentiating both sides with respect to $k$, one obtains

$$
\frac{k S(k) E(k a)}{\mu 4 \pi}=-\frac{d}{d k} W_{n a}(k \mu)
$$

Norton [7] and Wheelon [4] each obtained the result

$$
\frac{k S(k)}{\mu 4 \pi}=-\frac{d}{d k} W_{n}(k \mu)
$$

which, when combined with (27) yields

$$
\frac{d W_{n}(k \mu)}{d(k \mu)}=\frac{1}{E(k a)} \frac{d W_{n a}(k \mu)}{d(k \mu)}
$$

which relates the slope of the actual spectrum to the slope of the measured spectrum.

\section{Discussion of Results}

Because no data are available for calculating the wave number spectrum, the interpretation of results will be confined to the frequency spectrum. It is well to bear in mind, however, the assumptions made (eqs (23) and (24)) to obtain these results.

Referring to figure 2 and figure 4 it is seen that $E(k a) \simeq 1$ for $k a<0.5$, so that in this range the filter factor has very little effect. If one assumes that $a=2.5$ centimeters, which corresponds 
to a microwave refractometer cavity [2], it follows that $k<0.2$ per centimeter. For the constant wind velocity $\vec{\mu}$ of 5 meters per second (approximately $11 \mathrm{mph}$ ) this yields $f \leq 100 / 2 \pi$ cycles per second as the range in which the frequency spectrum is unaffected by the filter factor. The available data [8] gives a spectrum accurate to frequencies of at most 10 cycles per second, and the filter factor does not alter the spectrum in this range.

If the filter factor does go to zero, as in figure 2 and figure 4 , the first zero determines a definite upper limit for $k$ (or $f$ ) for which the refractometer determined spectrum is valid. However, refractometer measurements are generally inaccurate because of noise, etc., before this limit is realized.

The need for more data to determine the weighting function and the two spectra is apparent. Even if such data were available, it is felt that one of the three forms derived bere would give a satisfactory approximation.

\section{References}

[1] A. D. Wheelon, Radiowave scattering by tropospheric irregularities, J. Research, NBS, 63D, 205 (1959).

[2] G. Birnbaum and H. E. Bussey, Amplitude, scale and spectrum of refractive index inhomogeneities in the first 125 meters of the atmosphere, Proc. I.R.E., 43, 1412 (1955).

[3] M. C. Thompson, private communication.

[4] A. D. Wheelon, Relation of radio measurements to the spectrum of tropospheric dielectric fluctuations, J. Appl. Phys. 28, 684 (1957).

[5] Tables of integral transforms, Bateman Manuscript Project, Vol. 1, (McGraw-Hill Book Co., New York, N.Y., 1954).

Boulder, Colo.
[6] Radio studies of atmospheric turbulence (to be published).

[7] K. A. Norton, Point-to-point relaying via the scatter mode of tropospheric propagation, I.R.E. Trans. on Commun. Systems, CS-4, No. 1, 39 (1956).

[8] K. A. Norton, Recent experimental evidence favoring the $\rho K_{1}(\rho)$ correlation function for describing the turbulence of refractivity in the troposphere and stratosphere (to be published in the Proc. of the Joint Comm. on Radio Meteorology held under the auspices of the Intern. Sci. Radio Union at New York Univ., August, 1957).

(Paper 64D1-40) 